Revista Mexicana de Astronomía y Astrofísica, Volumen 3, Mhyo 1977

\title{
A NEW MICHELSON STELLAR INTERFEROMETER
}

\author{
R. Q. Twrss and W. J. Tango \\ Royal Observatory Edinburgh \\ (Presented by W. McD. Napier)
}

\begin{abstract}
RESUMEN
Un nuevo interferómetro con $1.87 \mathrm{~m}$ de línea de base fija, diseñado para observaciones de estrellas binarias está ahora en operación en la estación italiana del Observatorio Real de Edinburgo. Este equipo tiene un poder de resolución teórico de 0.015 seg. de arco y una magnitud B límite de 6.5. Se ha corregido dinámicamente el "seeing" atmosférico, permitiendo así, una medición cuantitativa de la visibilidad de franjas.
\end{abstract}

\section{ABSTRAGT}

A new $1.87 \mathrm{~m}$ fixed-baseline interferometer designed for binary star observation is now in operation at the Italian outstation of the Royal Observatory Edinburgh. It has a theoretical resolving power of 0.015 arcsec and a limiting $B$ magnitude of 6.5. Atmospheric seeing is dynamically corrected, allowing a quantitative measurement of fringe visibility.

We describe here a two-meter baseline Michelson stellar interferometer which has been built at the Italian out-station of the Royal Observatory Edinburgh, under the sponsorship of the British Science Research Council. Construction of the interferometer began in 1972 and the first interferometric stellar observations were made in June of this year (1975). At present the machine is undergoing its final commissioning and double star observations will begin this winter.

The instrument differs in several important respects from earlier Michelson stellar interferometers. First of all it has been designed specifically for double star observations, and has a resolving power of about 0.015 arcsec, equivalent to a diffraction limited 150 inch telescope. A second feature is that, because of the electronic detection techniques employed, there is no need for highly skilled observers as has been the case with visual interferometers in the past. Finally we note that because the instrument does not make use of a large aperture telescope, it is relatively inexpensive, costing approximately one percent as much as a telescope of equivalent resolution.
The interferometer is located $30 \mathrm{~km}$ southeast of the city of Rome, at a height of about $400 \mathrm{~m}$. It is housed in an observing tower, while the observer remains in an adjacent control room at ground level, from which the interferometer is remotely controlled. The specifications of the instrument are given in Table 1. The baseline and operating wavelength fix the resolution at about 0.015 arcsec, while the limiting magnitude of 6.5 (B) is determined by the apertures. Because of "seeing" it is necesary to restrict these to relatively small diameters, and in the present instrument they are $45 \mathrm{~mm}$.

To be of any astronomical value, an interferometer must be capable of quantitative measurements of fringe visibility, and our aim is to attain one percent accuracy in determining the visibility. To achieve this, it is necessary to remove the effects of atmospheric turbulence ("seeing") by a servo system. This desired accuracy additionally puts a constraint on the maximum path length errors which can be tolerated. For the given bandwidth, we require that the internal paths be equal to within about $25 \mu \mathrm{m}$. The dual requirements of active "seeing" correction 
TABLE 1

INTERFEROMETER SPEGIFICATION

\begin{tabular}{lll}
\hline Baseline Length: & 1.87 & meter \\
Aperture Diameter: & 45 & $\mathrm{~mm}$ \\
Wavelength: & 440 & $\mathrm{~nm}$ \\
Bandwidth: & 0.4 & $\mathrm{~nm}$ \\
Resolution: & 0.015 & $\operatorname{arcsec}$ \\
Limiting Magnitude (B): & 6.5 & \\
Maximum $\Delta \mathrm{m}:$ & 5 & \\
\hline
\end{tabular}

and high stability largely determined the final design of the instrument, which is shown schematically in Figures 1 and 2.

Figure 1 illustrates the altazimuth mounting of the interferometer. This configuration was chosen primarily because it provides an extremely stable mounting for the interferometer optics. The pointing accuracy is about 1 arcsec; this is achieved by a guidance system incorporating a desk top calculator and photoelectric autoguiders.
Figure 2 is a highly schematic outline of the main optical components of the instrument. Starlight enters the instrument via the elevation flats, $E$. Part of the starlight is focussed by the guide telescope, $T g$, onto "optical pyramids", $p y$, which act as image dissectors. The outputs are detected photoelectrically and used for the table and mirror guidance systems.

The main telescopes, $T$, bring the light into the interferometer proper. The path compensator, $C$, is used to obtain equality in optical paths (determined by finding white light fringes). Polarizing beam splitters, $b$, resolve the starlight into its $s$ and $p$ components; the $p$ polarization is used in the interferometer, while the $s$ polarized light is deflected into the anti-seeing system. This consists of long focus telescopes $t$ and optical pyramids; the outputs are used to stabilize the stellar image on the appropriate pyramid by means of a servo which controls tilting plates located in the main telescope $(T)$.

The light interferes in the beamsplitter $B_{o}$, and is filtered by the prism spectrometers $s p$. The two

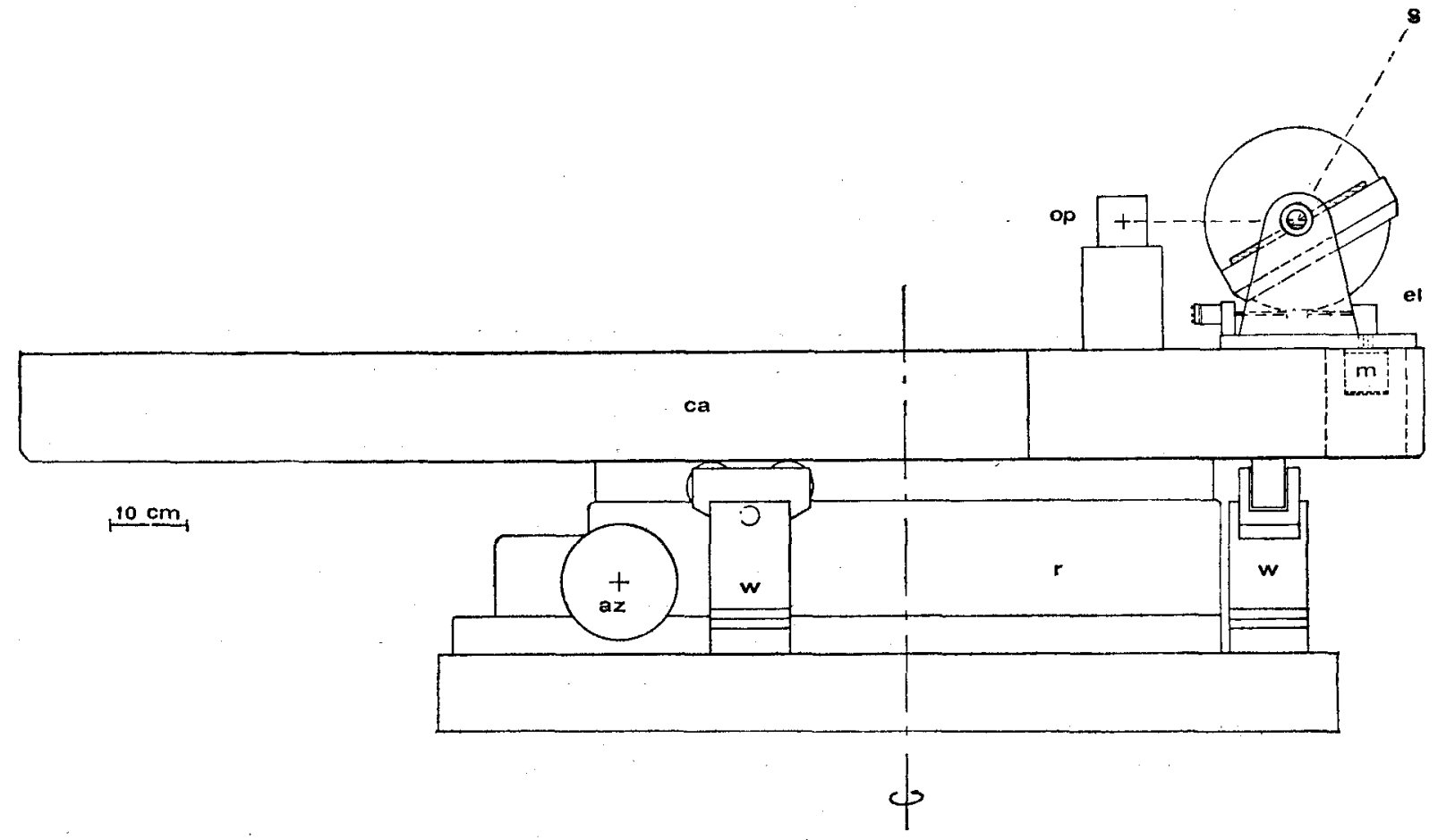

FIg. 1. Schematic of the Altazimuth Mounting. The chief components are: $r$ : precision rotary table; az: azimuth drive motor; $w$ : weight relieving devices to bear the weight of the main casting $c a$; $e l$ : the elevation mirror assembly (only one shown), driven by the motor $m$, which directs light from the star ( $S$ ) into the optical system (op). 


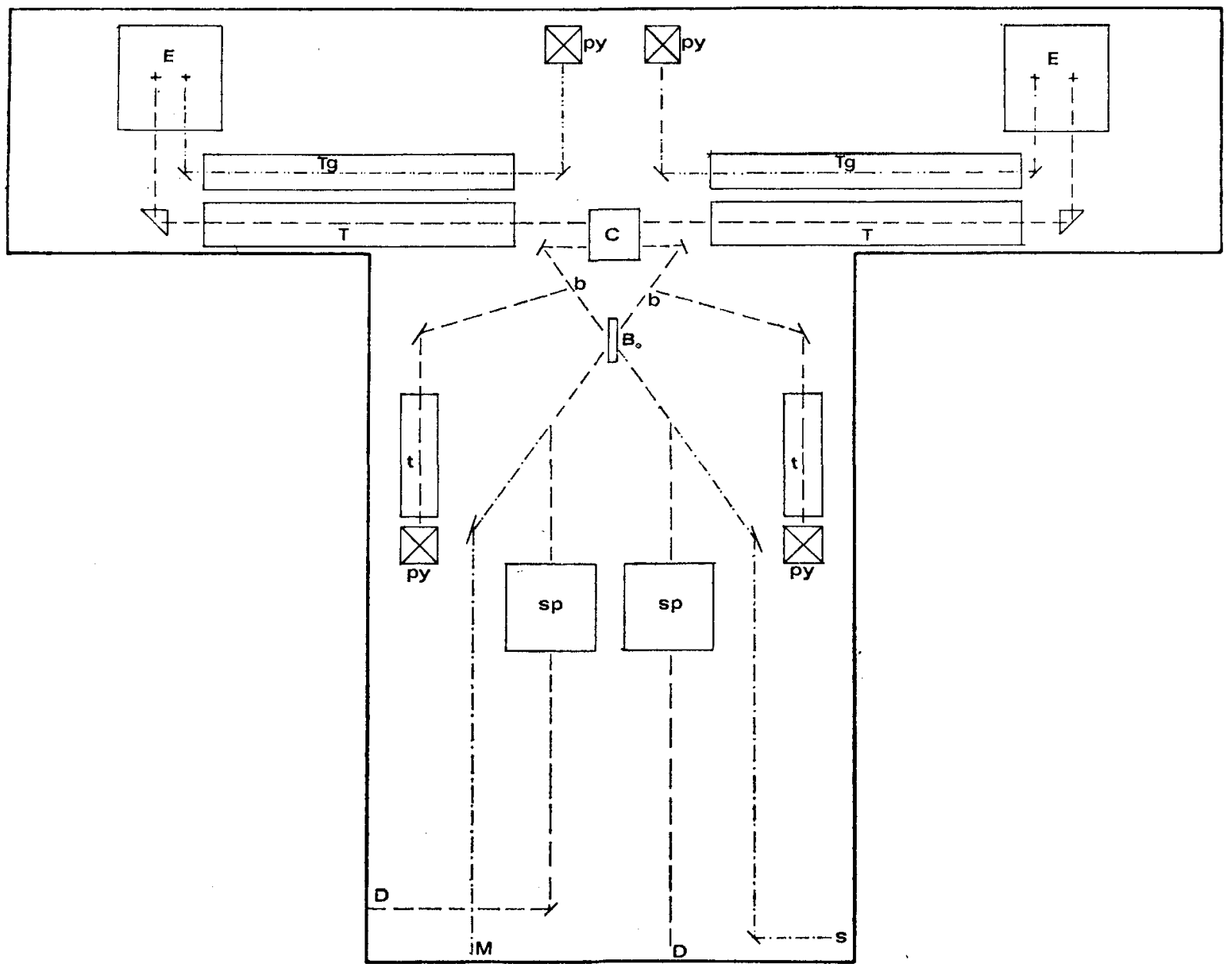

Fio.2. Idealized representation of the interferometer optics. See text for explanation of symbols.

interfering beams are then detected by the Channeltron photon counters located at $D$. The data is processed by a small special purpose calculator and recorded.

In addition to the interferometer optics, there are also several auxiliary optical systems which are used for aligning the instrument. Indeed, all of the necessary alignments can be performed during the daytime using artificial sources. Two such systems are indicated. The point $M$ permits the visual monitoring of the interferometer, and $s$ is an artificial star which is used with the instrument in a TwymanGreen configuration.

Because of the automatic data acquisition, as well as the optical and mechanical layout, it should be possible to detect duplicity or otherwise, quite rapidly. Consequently the interferometer is well suited for statistical or discovery programs, especially in the range $0.2-0.02$ arcsec. This is getting into the range of wide spectroscopic binaries, and hopefully will lead to cooperative efforts with spectroscopic observers on certain systems.

\section{DISCUSSION}

Beardsley: What estimate of $\Delta \mathrm{m}$ do you expect to achieve?

Napier: We expect to find duplicity with up to 5 magnitudes difference between com-

ponents. The accuracy obtained for $\Delta \mathrm{m}$ will probably not be high. 